\title{
Comparison and implementation of two methods in a truss optimization, one based in an evolutionary structural optimization, other based in mathematical programming.
}

\author{
Luiz P. R. Filho (IC), Renato Pavanello (PQ).
}

\begin{abstract}
This paper compares two different methods of a truss optimization. The goal of the optimization is to minimize the compliance of the truss like structures, maximizing the stiffness. The methods used are the Evolutionary Structural Optimization, which is based in slowly removing inefficient material of the structure ${ }^{1}$, and the Method of Moving Asymptotes, method developed by Kristen Svanberg, based in creating a strictly convex approximated subproblem ${ }^{2}$, which is solved in each iteration of the optimization process.
\end{abstract}

Key words: Truss, Optimization, Mathematical.

\section{Introduction}

In this work we optimize the compliance of a truss structure subject to a volume constraint, using topology optimization techniques. Several optimization methods have been developed based in continuous and discrete approximations. Among others techniques, Evolutionary Structural Optimization (ESO) has been consolidated in the area through the successive application of heuristic strategies. This method is based on a rule of successive elimination of elements in a finite element mesh that covers the feasible solution space defined initially. In this work, the chosen problem is the minimization of the compliance of truss structures. The initial guess solution is based on a fine mesh, considering a broad design space. Bars connecting all nodes are created, forming the initial solution. Then, a Finite Element analysis will be carried out ${ }^{3}$ and a sensitivity analysis ${ }^{1}$ is performed. With this sensitivity information, the two methods are applied: ESO and MMA. The design variable is the density of the volume of the bars. The ESO work taking the volume of the chosen bars to a minimum prescribed, and the MMA, on the other hand, allows the volume to be any number between the maximum and minimum limits. In other words, the ESO is a discrete optimization technique and the method based on the MMA is a continuous based technique. Both algorithm are implemented and tested in a Matlab program, and the obtained results are compared.

\section{Results and Discussion}

To illustrate, one truss with displacement constraint in the two low extremities and a force in the low middle is considered, see Figure 1. The results obtained using ESO and MMA methods are shown in the Figure 1. In the initial guess solution, all bars have the same volume/diameter. The global volume will decrease and the bars will take the optimum size according to the method. The convergence criteria is an average value of the last nine values of the compliance.

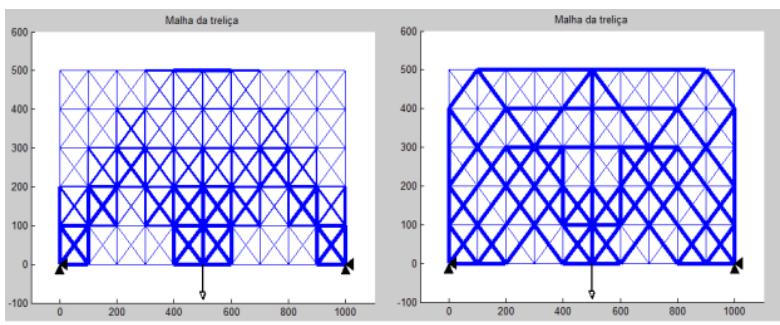

Figure 1. Result for MMA at left and for ESO at right.

Table 1. Comparison between the value of the compliance and the number of iterations.

\begin{tabular}{|c|c|c|}
\hline & MMA & ESO \\
\hline Compliance(Nmm) & 127,39 & 95,14 \\
\hline Iterations & 58 & 80 \\
\hline
\end{tabular}

Conclusions

The ESO method gets a smaller compliance, but with a higher number of iterations. The final obtained topologies are quite different but, both are similar with compared to previous results found in the literature other literature results.

\section{Acknowledgement}

I would like to thank Professor Svanberg for the brilliant code MMA.

\footnotetext{
${ }^{1}$ Huang, X.; Xie, Y. M.; Evolutionary Topology Optimization of Continuum Structures. 2010, 228p.

${ }^{2}$ Svanberg, K.; MMA and some modelling aspects. 1999, 8p.

${ }^{3}$ Kwon, Y. K.; Bang, H.; The Finite Element Method using MATLAB. 1997, 527p.

${ }^{4}$ Bendsoe, M. P.; Sigmund, O.; Top. Opt. 2002, 527p.
} 